\title{
A Survey of Music Personalized Recommendation System
}

\author{
Yuan $\mathrm{Xie}^{\mathrm{a})}$ and Lei Ding \\ School of Computer, Guangdong University of Technology, Guangdong 510006, China \\ a) Corresponding author: 944738579@qq.com
}

\begin{abstract}
The study of music recommendation research was conducted and the corresponding research hotspots were proposed. The literature analysis method was used to briefly introduce the recommendation strategy from the perspective of the recommendation algorithm. It further proposes a method of using rough set theory to extract important context information and combines this user preference in context with collaborative filtering recommendation technology to implement a new idea of context recommendation-based music recommendation. The existing studies have a lack of systematic research on user behaviors and needs, low levels of feature extraction, and single problems in evaluation indicators. In the future, more in-depth discussions can be conducted in terms of group music recommendation, ontology modeling, and contextual awareness-based music recommendation in a mobile environment.
\end{abstract}

Key words: literature analysis; recommendation; ontology; contextual awareness.

\section{INTRODUCTION}

With the rapid development of mobile networks and digital multimedia technologies, digital music has become the mainstream consumer content sought by many young people. Personalized music recommendation has also become a hot topic in the recommendation field. However, the huge amount of heterogeneous music data it generates undoubtedly surpasses the basic needs and affordability of the audience, which in turn leads to information fatigue. Therefore, how to use music personalized recommendations to help users quickly and accurately obtain music tracks of interest in the vast music library becomes more and more important.

In recent years, music recommendation technology has attracted close attention from domestic and foreign scholars, resulting in a lot of research results. There have also been many well-known and personalized music stations, such as Pandora and Last.fm, well-known foreign websites, domestic Song Taste and Douban Radio, etc. The accuracy and coverage of the recommendation results are low, and lack of personalization often does not really satisfy the users. Compared with other types of recommended content such as movies, readings, books, etc., music recommendations have unique characteristics. If the recommended track can be infinitely looped, it can only be used as background music; the type of playing song is strongly associated with the user's mood. This requires that the music personalized recommendation system can effectively reflect and reflect the audience's personal preferences and situations. Adjustments to achieve personalized recommendations for different audience needs. Therefore, the music personalized recommendation system is a more complex system than the general recommendation system. It needs to comprehensively consider the needs of the user and combines the audio feature recognition and speech processing technologies in the multimedia field to complete the extraction of music features. Taking music recommendation as the research object is a special field in the personalized recommendation system, which has great research value and practical significance. 


\section{THE MUSIC PERSONALIZED RECOMMENDATION SYSTEM}

Music personalized recommendation system is usually composed of three parts: user preference model, music resource description and recommendation algorithm [1]. Among them, the description of music resources mainly includes the organization and management of music resources (songs, singers, albums, etc.). The music feature database is constructed by defining different levels of complexity and degree of abstraction to provide effective input data for the music recommendation model. Therefore, music resources are the foundation and key to forming music recommendations. Pachet [2] divided music resources into three categories - Acoustic Metadata, Editorial Metadata, and Cultural Metadata.

At present, in the related research of the music personalized recommendation system, the introduction is mainly focused on the recommendation strategy or the improvement of its algorithm, ignoring the important influence of the recommendation object on the recommendation system. The uniqueness and complexity of the music recommendation are mainly reflected in the music resource description module, which relates to the annotation of audio files, the semantic understanding of the terminal user, the use of acoustic descriptors for the segmented audio, and the like. This kind of music information processing the special nature of the recommendation system puts forward new requirements.

1. Based on Acoustic Metadata: Acoustic metadata refers to the acoustic properties of some of the underlying layers of the song extracted through audio signal analysis. The extraction of these underlying features requires a series of preprocessing of the audio, such as quantization, anti-aliasing filtering, pre-emphasis, windowing, Fourier transform, and the like. Among the commonly used music feature parameters are: frequency center, short-term average energy, zero-crossing rate, MFCC, bandwidth and so on. Due to the limitation of the ability to extract music features, and its feature dimension is large, the time complexity of the algorithm is high, and it is difficult to guarantee real-time online processing. These factors all lead to that music recommendations based on acoustic metadata cannot be fully applied and developed.

2. Based on Editorial Metadata: Editing metadata is described by some domain experts' subjective and objective views on music or adopts a collaborative mode, and the qualitative description data given by group users. It mainly includes song production information (such as songwriter, singer, age, language, album included), singer background, music type, expert annotation, or commentary. Its core is to establish appropriate links between music works and their associated attributes, applying basic organizational principles to music resources. At present, the established music databases established using the edit metadata include AMG (All Music Guide), CDDB (CD Database), Musicbrainz, etc. However, there is a lack of personalization, high labor costs, poor scalability, and there is still a certain difference from the true musical perception of users.

3. Based on Cultural Metadata: Whitman et al. [3] proposed a method for explicit processing of singer's free text meta information in the music recommendation engine, using keywords such as song titles, reviews, artist names, etc. to filter web page data, comparing 5 recommended singers and 5 random selections. The singers' average of similarities, and suppose that when the average of the five recommended singers is higher, the system is verified to be able to better implement the singer's recommendation. The cultural metadata of music is the data foundation of many musicbased personalized recommendation systems based on labels. Their general idea is to use the social tags provided by users to comprehensively evaluate the relevance of singers or songs. The label not only reflects the category and content characteristics of the music itself, but also reflects the user's preference for music, and has strong flexibility and openness. It also has some problems. On the one hand, the acquisition of cultural metadata requires the presence of a large number of highly viscous users; on the other hand, it is prone to prejudice, and songs on the long tail of music are ignored by the audience.

\section{THE HOT RESEARCH OF MUSIC PERSONALIZED RECOMMENDATION}

The following further researches and discussions have been conducted in terms of group music recommendation, ontology modeling, and context-aware music recommendation in a mobile environment.

1. Group music recommendation system: Although music personalized recommendation systems are rapidly developing in recent years, most systems currently only recommend for a single user, and music as an important way of emotional expression and emotional communication has significant public sociality. Therefore, consider establishing a music recommendation system that is suitable for the public environment. Realizing real-time recommendation services for group users will provide new research ideas for music recommendation. Popescu et al. [4] constructed a group music recommendation system, GroupFun, which uses a voting mechanism to express the true 
musical preferences of group users and uses a probability weighted sum approach to evaluate the final satisfaction of group users with respect to the recommended songs.

2. Ontology modeling: Ontologies have the characteristics of semantic multiplicity and excellent reasoning capabilities, which have been widely used in many contextual intelligence systems. Foreign scholars have tried to use ontology to model music to solve the semantic gap between low-level audio features and deeper user understanding of music. Music ontology has been specifically developed for music recommendation services [5], and on this basis, definitions have been made. Ontology related to music. Due to different cultural backgrounds and different ways of thinking, the music ontology for Western music and listeners does not apply to Chinese songs and Chinese-based music. Therefore, the construction of a user model based on the Chinese music-based music ontology or application ontology technology will become an important research hotspot.

3. Evaluation metrics: At present, most music personalized recommendation systems use RMSE, MAE, or a Top$\mathrm{N}$ recommendation list to measure recommendation results through Precision and Recall. However, regardless of which of the above metric methods is used, the essence is to use the prediction accuracy as an index for evaluating a music personalized recommendation system. Therefore, how to solve the problem that the current system generally has a single evaluation index becomes an important research hotspot. To address this issue, consider adding some new measurement indicators. Celma [6] proposes to use the average popularity of the recommendation results for evaluation. The basic idea is that the music that is at the end of the popularity of the popularity is more likely to make the user feel new. Assuming that $\mathrm{S}$ represents a set of users, the novelty definition of user u's recommendation list is as follows [7]:

$$
\text { Novelty }=\frac{1}{|S|} \sum_{u \in S} \sum_{i \varepsilon R R_{u, 20}} \frac{\log _{2} p o p_{i}}{20}
$$

Among them, $\mathrm{R}$ is a Top- $\mathrm{N}$ function, $\mathrm{Ru}, 20$ indicates that the top 20 items in the recommendation list are recommended to user $\mathrm{u}$, and popi indicates the preference estimate for the item.

The degree of surprise of music recommendation refers to a song that is not similar to the user's favorite song, but the user feels satisfied. For example, fans who recommend John Lennon's songs to a Beatles may be accurate and novel recommendations, but they are not a recommendation that can surprise users. Therefore, if the recommendation result is not similar to the user's past interest, but it is satisfied with the user, then it can be said that this recommendation result has a high degree of surprise. The degree of surprise is measured by Unserendipity [8]. The formula is as follows:

$$
\text { Unserendipity }=\sum_{u \in S} \frac{1}{|S|\left|H_{u}\right|} \sum_{h \in H_{u}} \sum_{i \varepsilon R_{u, 20}} \frac{\operatorname{CosSim}(\mathrm{i}, \mathrm{h})}{20}
$$

Among them, the CosSim(i,h) function is to compare the average similarity between the user's historical interest $\mathrm{Hu}$ and the new recommendation result. The smaller the value, the more deviating the recommendation result from the user's historical interest, and the degree of surprise of the recommendation result. The higher it is.

4. Personalized recommendation system based on context awareness in mobile environment: With the popularization of smart mobile terminals and Location Based Service (LBS) in recent years, users request music recommendation services through mobile terminals become a new trend, and music applications based on smart mobile terminal devices are also receiving increasing attention. The current music recommendation system mainly relies on static User Profiles for music recommendation, which often leads to convergence of recommendation results for different users and lack of personalization. The main reason for this is the current music personalized recommendation system. All ignore the influencing factors that play a key role in user's music selection, namely the user's context [9], which mainly includes the time, location, mood, weather, current activity, etc. of the user's access to the recommendation system. When a person is happy and physically exhausted, the type of music they prefer is definitely different. When they are in a quiet country and a city that is in a hustle and bustle, the feeling of the same song must be different. Therefore, considering the user's contextual factors in the mobile environment and recommending music based on these specific contextual factors will certainly become a hot topic in the music recommendation field. Different from the traditional music recommendation service, the context recommendationbased music recommendation service in the mobile environment is completed on the mobile terminal. Since the current mobile device is highly intelligent and has strong user dependence, the mobile intelligent terminal can act as a sensor to capture the user in real time. Listen to the context information of the music, get a large number of real-time field data sets, and lay a good data foundation for music recommendations. However, mobile terminals also have some 
inherent flaws, such as limited display size and processing power. In addition, mobile devices should be used as interaction media with music recommendation systems, and care should be taken to protect the privacy of users while ensuring that the information is reasonable.

\section{CONCLUSION}

This article reviews the current music recommendation research results from the perspective of music resources description. It points out that there is a lack of systematic research on user behavior and needs, low level of feature extraction, and a single evaluation index in current research. At the same time, Group music recommendation, ontology modeling, context-aware music recommendation based on mobile environment, and other aspects of further research and discussion. Among them, the situation is considered to be an important factor in the music personalized recommendation system. However, in the current music personalized recommendation system, the weights given to all contextual factors are the same. Obviously, this kind of weight division is unreasonable, and it greatly reduces the accuracy of the recommendation results. Therefore, the idea of the next step is to use the rough set theory to reduce the attributes of the situation, calculate the weight of the different context attributes for the music recommendation, and extract the attributes that have a greater influence on the music recommendation (such as mood, current activities, and weather Etc.), and then incorporate it into the collaborative filtering recommendation algorithm. Through important situational similarity calculations and song similarity calculations, a list of music recommendations is generated for users in a specific situation, and context-aware music recommendation is realized.

\section{ACKNOWLEDGMENTS}

This work is supported by the national natural science foundation of China under grant (No. 61202267), natural science foundation of Guangdong province under grant (No.2016A030313713).

\section{REFERENCES}

1. Campbel M E, Clune Iii J E, Hicken W T, et al. Music Recommendation System and Method: USA, WO2005038666 A1[P]. 2004-08-13.

2. Pachet F. Knowledge Management and Musical Metadata[A]//Schwartz D. Encyclopedia of Knowledge Management[M]. Idea Group, 2005.

3. Whitman B, Lawrence S. Inferring Descriptions and Similarity for Music from Community Metadata [C]. In: Proceedings of the 2002 International Computer Music Conference, 2002: 591-598.

4. Popescu G, Pu P. What's the Best Music You Have: Designing Music Recommendation for Group Enjoyment in GroupFun [C]. In: Proceedings of the 2012 ACM Annual Conference Extended Abstract on Human Factors in Computing Systems Extended Abstract. ACM, 2012: 1673-1678.

5. Raimond Y, Abdallah A S, Sandler M, et al. The Music Ontology [C]. In: Proceedings of the 8th International Conference on Music Information Retrieval. 2007: 417-422.

6. Celma Ò. Music Recommendation [A]// Music Recommendation and Discovery [M]. Springer Berlin Heidelberg, 2010:43-85

7. Hurley N, Zhang M. Novelty and Diversity in Top-N Recommendation-Analysis and Evaluation [J]. ACM Transactions on Internet Technology, 2011, 10(4): Article No.14.

8. Murakami T, Mori K, Orihara R. A Method to Enhance Serendipity in Recommendation and Its Evaluation [J]. Transactions of the Japanese Society for Artificial Intelligence, 2009, 24(5): 428-436.

9. Adomavicius G, Tuzhilin A. Context-Aware Recommender Systems [A]// Recommender Systems Handbook [M]. Springer US, 2011: 217-253. 